\title{
Synchronous double primary malignancies of the liver and kidney: A case report
}

\author{
JUN-JUN SUN, TIAN-BAO YANG, YAN-HUI YANG, WEI-FENG LIU and JUN-XIN SONG \\ Department of General Surgery, The First Affiliated Hospital of Henan University of Science and Technology, \\ Luoyang, Henan 471003, P.R. China
}

Received December 7, 2014; Accepted January 14, 2016

DOI: $10.3892 / \mathrm{ol} .2016 .4194$

\begin{abstract}
A 42-year-old male patient was admitted to the Department of General Surgery of The First Affiliated Hospital of Henan University of Science and Technology (Luoyang, China) presenting with abdominal discomfort. Enhanced computed tomography of the abdomen revealed a $15.1 \times 7.0-\mathrm{cm}$, enhanced, double-spherical, exogenous, solid tumor originating from the left lateral hepatic lobe, in addition to a $4.3 \times 4.2-\mathrm{cm}$ mass in the mid portion of the left kidney. Pre-operative imaging analysis resulted in the diagnosis of double cancer, consisting of hepatocellular carcinoma (HCC) and renal cell carcinoma (RCC). The patient subsequently underwent left hemihepatectomy and left nephrectomy. Histological examination confirmed that the tumor originating from the left lateral hepatic lobe was HCC, and the tumor arising from the mid portion of the left kidney was clear cell RCC (ccRCC). The post-operative follow-up was uneventful. To the best of our knowledge, the present case is the first of its kind to describe the resection of synchronous double cancer, consisting of primary HCC and ccRCC.
\end{abstract}

\section{Introduction}

The increased survival of patients with cancer, the growing life expectancy and the development of improved diagnostic techniques have all contributed to the increased frequency of multiple primary malignancies (1). In a number of cases, it is difficult to differentiate between two primary neoplasms or metastatic diseases, although the distinction is significant as the staging, further management and prognosis are completely different (2). The distinction between metastatic

Correspondence to: Professor Jun-Jun Sun, Department of General Surgery, The First Affiliated Hospital of Henan University of Science and Technology, 24 Jinghua Road, Luoyang, Henan 471003, P.R. China

E-mail: doctsunjj@sina.com

Key words: hepatocellular carcinoma, renal clear cell carcinoma, synchronous double primary malignant tumors, hepatectomy, nephrectomy and independent tumors is also notable since it affects staging and prognosis differently (2). In the present case, the malignant features of each tumor were synchronously confirmed by post-operative pathological diagnosis.

Currently, there is no universally accepted, standard treatment for multiple primary malignancies. The treatments of choice, depending on the tumor location, include curative surgical resection of each malignancy, radiotherapy and chemotherapy (3). The most common treatment is surgery associated with adjuvant treatment (3). The treatment of synchronous double cancer generally relies on surgery; however, in surgically non-resectable tumors, chemotherapy is considered the most promising form of treatment, targeting each tumor, but concentrating on the most aggressive (4). In the present case, the treatment of choice was curative resection of each lesion, and the post-operative course was uneventful. The patient is followed-up regularly, with clinical examinations and liver function tests implemented every 2 months. Additionally, tumor markers [including $\alpha$-fetoprotein (AFP), carbohydrate antigen (CA)19-9, CA 125 and carcinoembryonic antigen (CEA)] have been monitored and abdominal computed tomography (CT) scans have been performed every 3 months. The patient is currently healthy, with no evidence of local or distant recurrence post-surgery.

\section{Case report}

A 42-year-old male patient was referred to the Department of General Surgery of The First Affiliated Hospital of Henan University of Science and Technology (Luoyang, China) in August 2014, presenting with poor appetite and abdominal discomfort in the right upper quadrant for 3 months. In 2004, the patient had been diagnosed with chronic liver disease secondary to hepatitis B at The People's Hospital of Yiyang County (Luoyang, China). In addition, the patient had a previous history of alcohol abuse for 17 years (alcohol intake, $250 \mathrm{~g} /$ day) and had smoked for 20 years. There was no remarkable family history. On admission, the vital signs were all within the normal ranges (heart rate, 80 beats/min; normal range, 60-100 beats/min; blood pressure, $130 / 70 \mathrm{mmHg}$; normal range, $60 / 90-80 / 140 \mathrm{mmHg}$, body temperature, $36.5^{\circ} \mathrm{C}$; normal range, $36-37.3^{\circ} \mathrm{C}$ and respiration rate, 20 times $/ \mathrm{min}$; normal range, $12-20$ times/min). The patient was generally in good health and did not exhibit any significant weight loss. 


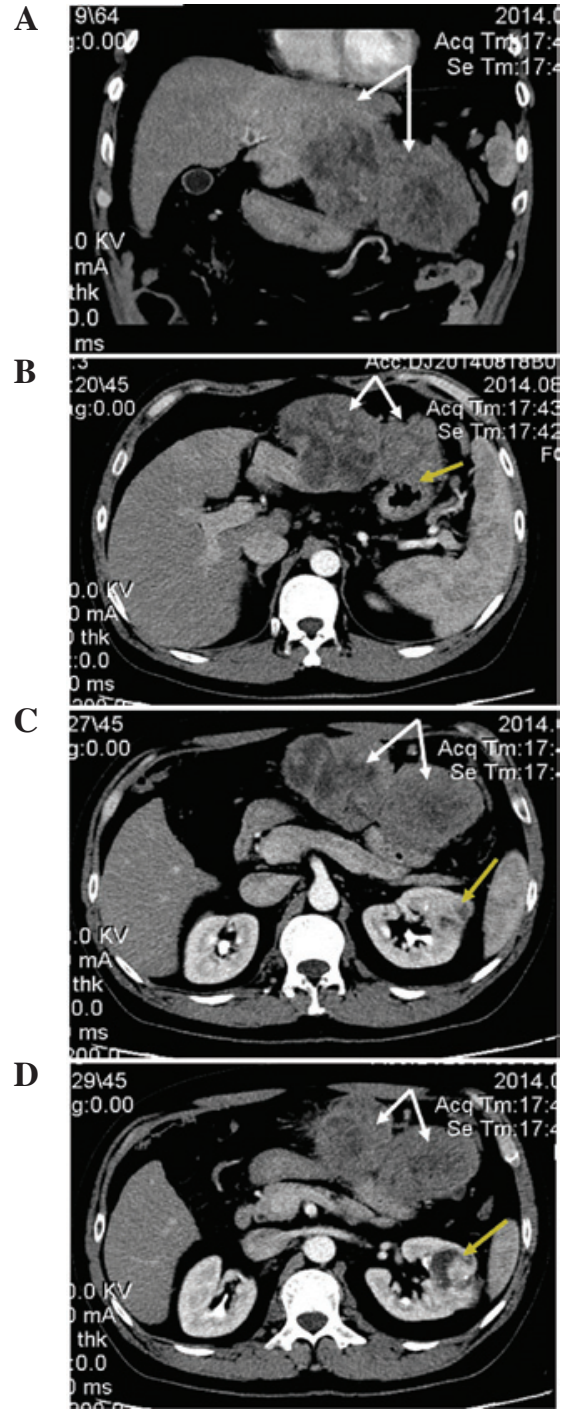

Figure 1. Enhanced computed tomography images. A large, low-density, double-spherical, exogenous, solid tumor originating from the left lateral hepatic lobe with a slightly heterogeneous enhanced peripheral rim (white arrows) exhibited on (A) coronal and (B) horizontal sections. The tumor had invaded the antrum of the stomach (yellow arrow). (C and D) Secondary tumor presenting as a mild, heterogeneous, enhanced, mixed-density mass in the mid portion of the left kidney (yellow arrow). The tumor originating from the left lateral hepatic lobe is indicated by the white arrows.

On physical examination, the conjunctiva was normal. The abdomen was soft, but tender in the right upper quadrant; it was noted that there was light resistance in this area, but no rigidity. The pre-operative serum biochemistry and complete blood count data on admission were as follows: White blood cell count, $6.62 \times 10^{9}$ cells/1 (normal range, 4.00-10.00×10 ${ }^{9}$ cells/1); hemoglobin count, $132.00 \mathrm{~g} / 1$ (normal range, $110.00-160.00 \mathrm{~g} / 1)$; platelet count, $225.00 \times 10^{9}$ cells $/ 1$ (normal range, 100.00-300.00 $\times 10^{9}$ cells/1); blood glucose, $5.20 \mathrm{mmol} / 1$ (normal range, 3.90-6.00 $\mathrm{mmol} / \mathrm{l}$ ); total bilirubin, $17.30 \mu \mathrm{mol} / 1$ (normal range, $0.00-20.00 \mu \mathrm{mol} / 1$ ); aspartate transaminase, $26.00 \mathrm{U} / 1$ (normal range, 15.00-45.00 U/1); alanine transaminase, $31.00 \mathrm{U} / 1$ (normal range, 9.00-50.00 U/1); alkaline phosphatase, $40.00 \mathrm{U} / 1$ (normal range, 30.00-150.00 U/1); lactic dehydrogenase, $183.00 \mathrm{U} / 1$ (normal range, 90.00-245.00 U/1); serum urea, $5.70 \mathrm{mmol} / 1$ (normal range, 2.60-6.30 mmol/1); serum creatinine, $118.00 \mu \mathrm{mol} / 1$ (normal

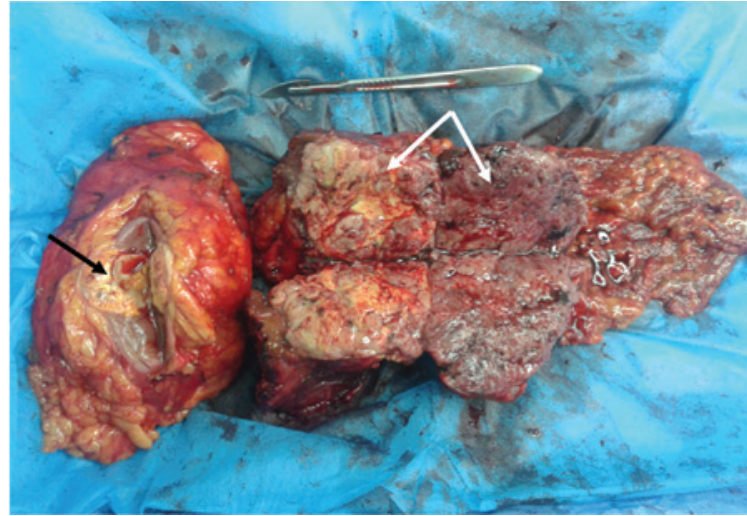

Figure 2. Resected surface of the tumors showing a yellow-white, solid tumor measuring 4.0x4.0x4.0 cm originating from the left kidney (black arrow), and a yellow and light brown, double-spherical, exogenous, solid tumor measuring $15.0 \times 8.0 \times 7.0 \mathrm{~cm}$ originating from the left lateral lobe of the liver (white arrows).

range, 40.00-110.00 $\mu \mathrm{mol} / \mathrm{l}$ ); albumin, $34.80 \mathrm{~g} / 1$ (normal range, 40.00-55.00 g/l); fibrinogen, $4.67 \mathrm{~g} / 1$ (normal range, 2.00-4.00 g/l); prothrombin time, $14.20 \mathrm{sec}$ (normal range, 11.00-15.00 sec); prothrombin time international normalized ratio, 1.19 (normal range, 0.80-1.20); AFP, 6,050.00 ng/ml (normal range, 0.00-7.00 ng/ml); CEA, $1.86 \mathrm{ng} / \mathrm{ml}$ (normal range, 0.00-4.30 ng/ml); CA 19-9, $5.43 \mathrm{U} / \mathrm{ml}$ (normal range, 0.00-27.00 U/ml); and CA 125, $36.70 \mathrm{U} / \mathrm{ml}$ (normal range, 0.00-36.00 U/ml). The viral markers were as follows: Hepatitis B surface antigen, positive; hepatitis B core antibody, positive; hepatitis Be antibody, positive; and anti-hepatitis $\mathrm{C}$ virus, negative.

An enhanced CT [TSX-301A; Toshiba Medical Systems Corp., Tokyo, Japan; contrast medium, iopromide (Bayer AG, Leverkusen, Germany)] scan of the abdomen revealed a $15.1 \times 7.0-\mathrm{cm}$, irregularly-enhanced rim, double-spherical, exogenous, solid tumor originating from the left lateral hepatic lobe (Fig. 1), which invaded the antrum of the stomach (Fig. 1B). The CT scan also revealed a $4.3 \times 4.2-\mathrm{cm}$, mildly-enhanced, mixed-density mass in the mid portion of the left kidney (Fig. 1C and D).

The pre-operative diagnosis was HCC and RCC. A laparotomy was performed, during which a large dumbbell-shaped lesion, which originated from the left lateral hepatic lobe, was observed to occupy the epigastrium and have invaded the antrum of the stomach. The patient underwent left hemihepatectomy and partial gastrectomy, in addition to left nephrectomy. Intraoperative histological examination revealed the presence of HCC, which had invaded the gastric antrum, alongside RCC. The patient was subsequently diagnosed with synchronous double primary cancer of the liver and kidney.

The resected surface of the tumor in the left kidney revealed a yellow-white, solid lesion that measured $4.0 \times 4.0 \times 4.0 \mathrm{~cm}$ in size (black arrow, Fig. 2). There was a yellow and light brown, double-spherical, exogenous, solid tumor that measured $15.0 \times 8.0 \times 7.0 \mathrm{~cm}$ in size and originated from the left lateral lobe of the liver (white arrows, Fig. 2).

For microscopic examination, the gross samples were sectioned as a tissue mass measuring $1.5 \times 1.5 \times 0.2 \mathrm{~cm}$ in size, and were fixed in $10 \%$ formalin at room temperature for $24 \mathrm{~h}$. 

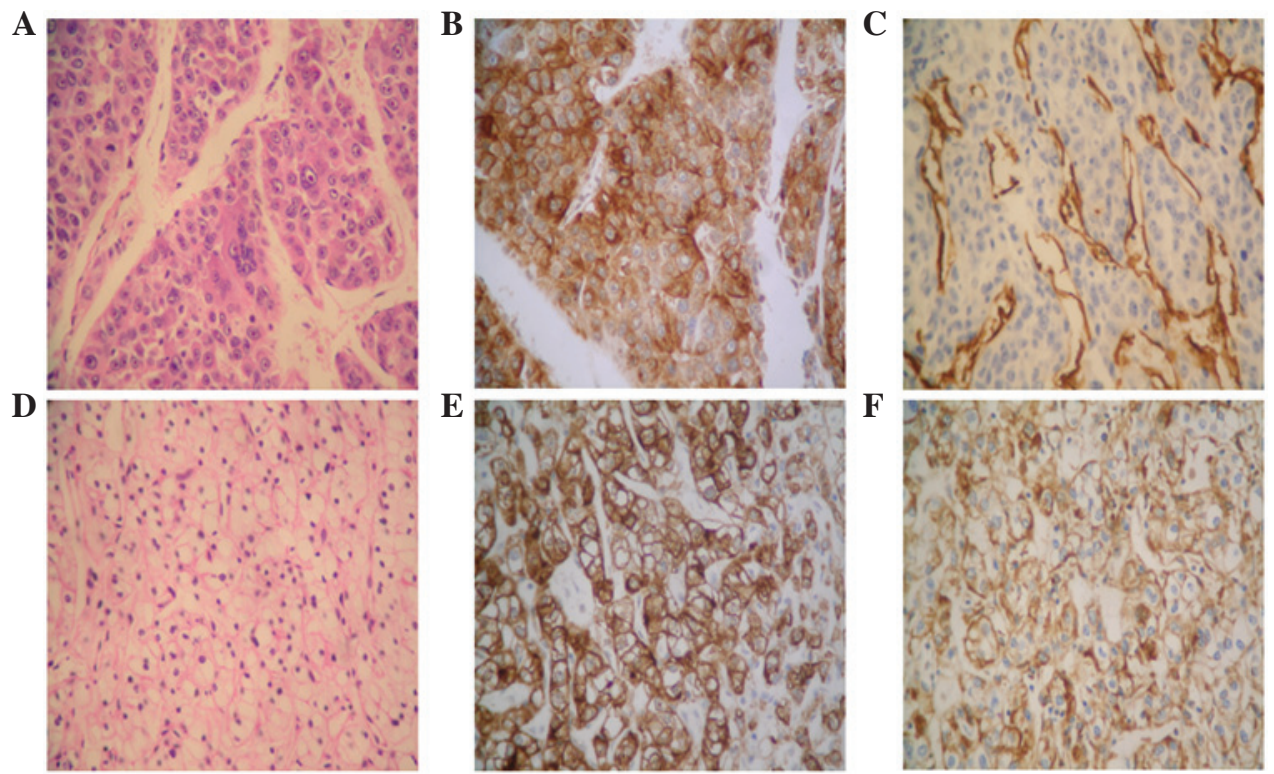

Figure 3. (A-C) Histological analysis of the resected HCC tumor tissues. (A) Poorly-differentiated HCC exhibiting cell clusters with a flake-like distribution, which were separated by a sinusoid blood vessel. The dividing line of the malignant cells was not clear, and their cytoplasm was eosinophilic. In addition, the cells exhibited large, heteromorphic, eosinophilic nucleoli (staining, H\&E). (B) Brown/yellow color indicates positive staining for hepatocyte-specific antigens in the cytoplasm and cellular membrane. (C) Brown/yellow color indicates positive staining for CD34 in the vascular endothelial cells. (D-F) Histological analysis of the resected ccRCC tumor tissues. (D) Moderately-differentiated ccRCC. The tumor cells were hyperchromatic and presented diffuse distribution, transparent cytoplasm and karyopyknosis. The dividing lines of the malignant cells were clear (staining, H\&E). Brown/yellow color indicates positive staining for (E) CD10 and (F) epithelial membrane antigen in the cytoplasm and cellular membrane. Magnification, x400. HCC, hepatocellular carcinoma; H\&E, hematoxylin and eosin; CD, cluster of differentiation; ccRCC, clear cell renal cell carcinoma.

The samples were then processed through the Leica ASP300 S Fully Enclosed Tissue Processor (Leica Microsystems GmbH, Wetzlar, Germany) and paraffin-embedded by the Tissue Embedding System TES 99 (Medite GmbH, Burgdorf, Germany). The paraffin-embedded tissues were sectioned into $4 \mu \mathrm{m}$ slices ready for examination using the HM 325 Rotary Microtome (Thermo Fisher Scientific, Inc., Waltham, MA, USA). On microscopic examination, the tumor in the mid portion of the left kidney exhibited diffusely distributed tumor cells with transparent cytoplasm, karyopyknosis and hyperchromatism. The dividing lines of the malignant cells were clear and reacted positively to cluster of differentiation (CD)10 (mouse anti-human monoclonal antibody; catalog no., MAB-0668; Fuzhou Maixin Biotech Co., Ltd., Fuzhou, China) and epithelial membrane antigen (mouse anti-human monoclonal antibody; catalog no., Kit-0011; Fuzhou Maixin Biotech Co., Ltd.) by immunostaining. The tumor originating from the left lateral hepatic lobe exhibited cell clusters with a flake-like distribution, which were separated by a sinusoid blood vessel. The dividing line of the malignant cells was not clear, and their cytoplasm was eosinophilic. In addition, the cells exhibited large, heteromorphic, eosinophilic nucleoli. Immunostaining demonstrated a positive reaction for hepatocyte-specific antigens (mouse anti-human monoclonal antibody; catalog no., MAB-0249; Fuzhou Maixin Biotech Co., Ltd.) and CD34 (mouse anti-human monoclonal antibody; catalog no., Kit-0004; Fuzhou Maixin Biotech Co., Ltd.). The left kidney tumor was diagnosed as moderately-differentiated ccRCC, and the left hepatic lobe tumor was diagnosed as poorly-differentiated HCC (Fig. 3).

The post-operative course was uneventful, and the patient was discharged on day 22 post-surgery. Adjuvant chemoradiation therapy was advised, as the HCC tumor was particularly large (diameter, $>10.0 \mathrm{~cm}$ ) with adjacent viscera invasion. However, this was not administered, as the patient declined further treatment. The patient has been followed-up regularly with clinical examination and liver function tests implemented every 2 months. Additionally, tumor markers (including AFP, CA 19-9, CA 125 and CEA) have been monitored and abdominal CT scans have been performed every 3 months. The patient is currently healthy, with no evidence of local or distant recurrence post-surgery.

\section{Discussion}

The occurrence of multiple primary malignant tumors in a single patient is particularly rare, with a literature review of 1,104,269 patients with cancer reporting the incidence of multiple primary malignancies as $0.73-11.70 \%$ (5). The following diagnostic criteria have been proposed for the accurate diagnosis of multiple primary malignancies: i) Each tumor must be distinct; ii) each tumor must exhibit marked features of malignancy; and iii) the probability of one lesion being a metastasis of the other must be excluded (6). Multiple primary malignancies may be synchronous or metachronous, often depending on the length of the interval between diagnoses (7). Synchronous multiple malignancies are secondary lesions that present simultaneously or within 6 months following the development of the initial malignancy, while metachronous multiple malignancies are secondary lesions that present $>6$ months following the development of the initial malignancy (8). In the present case, histopathological analysis confirmed the malignant features of each tumor. The tumors were pathologically established as different types of cancer 
and had developed within different systems, with the tumor in the kidney confirmed as moderately-differentiated ccRCC and the tumor in the liver confirmed as poorly-differentiated HCC. These findings support the notion that these two types of cancer occurred in a random and synchronous manner. HCC is understood to be pathogenically associated with chronic hepatitis virus infection, abuse of alcohol and liver cirrhosis (9). Although the mechanisms underlying the occurrence of multiple primary malignancies are not fully understood, certain factors have been implicated, including genetic factors, carcinogenic viruses, immunological and environmental factors, and chemical and radiological treatments (10). With regards to the current case, chronic hepatitis $\mathrm{B}$ virus infection may have served a crucial role in the development of HCC. The prognosis of patients with multiple primary malignancies may be determined independently by the stage of each malignancy (11). In the present case, the treatment of choice was curative resection of each lesion.

To the best of our knowledge, the present case is the first of its kind to describe the occurrence of synchronous double primary cancer of the kidney and liver. When treating patients with malignant tumors, the possibility of developing a secondary primary malignancy should be considered. The incidence of multiple primary malignancies does not necessarily signify an unfavorable prognosis, as long as satisfactory diagnosis and effective treatment are performed.

In summary, the present case of synchronous double primary cancer of the kidney and liver was confirmed by pathological and immunohistochemical analyses. Imaging findings may be helpful to assist with achieving a correct preoperative diagnosis. If in doubt, intraoperative frozen section analysis may be necessary to select the correct surgical approach. Simultaneous removal of multiple primary cancers should be attempted, and adjuvant treatment (radio/chemotherapy) should also be considered. Healthcare workers should consider that the appearance of an additional tumor in a cancer patient may be either a metastatic or novel lesion, and the possibility of a metachronous or a synchronous malignancy should be investigated. Furthermore, prolonged follow-up after surgery should be performed.

\section{References}

1. Spratt JS Jr and Hoag MG: Incidence of multiple primary cancers per man-year of follow up: 20-year review from the Ellis Fischel State Cancer Hospital. Ann Surg 164: 775-784, 1966.

2. Sheu BC, Lin HH, Chen CK, Chao KH, Shun CT and Huang SC: Synchronous primary carcinomas of the endometrium and ovary. Int J Gynaecol Obstet 51: 141-146, 1995.

3. Irimie A, Achimas-Cadariu P, Burz C and Puscas E: Multiple primary malignancies - epidemiological analysis at a single tertiary institution. J Gastrointestin Liver Dis 19: 69-73, 2010.

4. Kourie HR, Markoutsaki N, Roussel H, Rahmi G, Van der Stiegel M, Palazzo L, Fabre M, Cuenod CA, Dubreuil O, Landi B, et al: Double pancreatic and gastric adenocarcinomas: A rare association. Clin Res Hepatol Gastroenterol 37: e137-e140, 2013.

5. Demandante CG, Troyer DA and Miles TP: Multiple primary malignant neoplasms: Case report and a comprehensive review of the literature. Am J Clin Oncol 26: 79-83, 2003.

6. Warren S and Gates O: Multiple primary malignant tumors: A survey of the literature and a statistical study. Am J Cancer 16: 1358-1414, 1932.

7. Suzuki T, Takahashi H, Yao K, Inagi K, Nakayama M, Makoshi T, Nagai $\mathrm{H}$ and Okamoto M: Multiple primary malignancies in the head and neck: A clinical review of 121 patients. Acta Otolaryngol (Suppl 122): 88-92, 2002.

8. Yun HR, Yi LJ, Cho YK, Park JH, Cho YB, Yun SH, Kim HC, Chun HK and Lee WY: Double primary malignancy in colorectal cancer patients - MSI is the useful marker for predicting double primary tumors. Int J Colorectal Dis 24: 369-375, 2009.

9. Ohwada S, Yoshihiro O, Iwazaki S, Tanahashi Y, Sawada T, Takeyoshi I, Kawashima Y, Nakaura S, Iino Y and Morishita Y: Double cancer in different hepatic lobes: Hepatocellular and cholangiocellular carcinoma. Hepatogastroenterology 42: 411-414, 1995.

10. Tamura M, Shinagawa M and Funaki Y: Synchronous triple early cancers occurring in the stomach, colon and gallbladder. Asian J Surg 26: 46-49, 2003.

11. Yoshino K, Asanuma F, Hanatani Y, Kumai K and Ishibiki K: Statistical studies on multiple primary cancers including gastric cancers. Gan No Rinsho 30 (Suppl 12): S1514-S1523, 1984 (In Japanese). 